\title{
Breast implants: a rare cause of anaplastic large T-cell lymphoma
}

\section{SAINT-LUC UCL BRUXELLES \\ S. Bailly, S. Amat, R. Dehon, Y. Breners, L. Maindiaux, F. Dall Armenia, A. Capes, E. Van Den Neste, A. Camboni, C. Galant, M.-C. Vekemans.}

\section{Department of Hematology and Pathology, Cliniques universitaires Saint-Luc, Woluwé-saint-Lambert, Belgium}

\begin{abstract}
Primary lymphomas of the breast are extremely rare and most often are of B-cell origin. The first case of breast implant anaplastic large T-cell lymphoma (BI-ALCL) was reported in 1997. Several risk factors are suggested, but the underlying causes remains unclear. BIALCL can present as a late periprosthetic effusion (most common manifestation), an effusion in combination with a palpable mass, a breast mass alone, or without a seroma or mass and only detectable lymph node involvement. Ultrasound and magnetic resonance imaging are the best imaging modalities for detecting effusion. The diagnosis is made by cytologic analysis, and histological sample is recommended when a solid mass is detected. In contrast to lymphomas, BI-ALCL is often curable with surgery alone. The mainstay of treatment is complete removal of the prosthesis and the capsule with negative margins.
\end{abstract}

We report the case of a 45-year-old woman with a Li-Fraumeni syndrome who was diagnosed 17 years ago for a left breast carcinoma treated by chemotherapy, radiotherapy and mastectomy. Breast reconstruction had been performed 16 years ago with sub-cutaneous placement of silicone breast implant. Eleven years after, she develops a breast carcinoma in the right breast and was treated in the same manner. The patient complained of left breast isolated oedema without any history of pain, trauma, prodromal illness, local sign of infection, night sweats, weight loss, poor appetite or fever. On physical examination breast asymmetry was palpable. The left breast was distended firm and sensitive. The right breast was normal. No signs of local infection were observed. Initial blood analysis, including blood count, C-reactive protein, chemistry, lactate dehydrogenase and blood culture were all normal. A puncture was normal, without sign of infection.

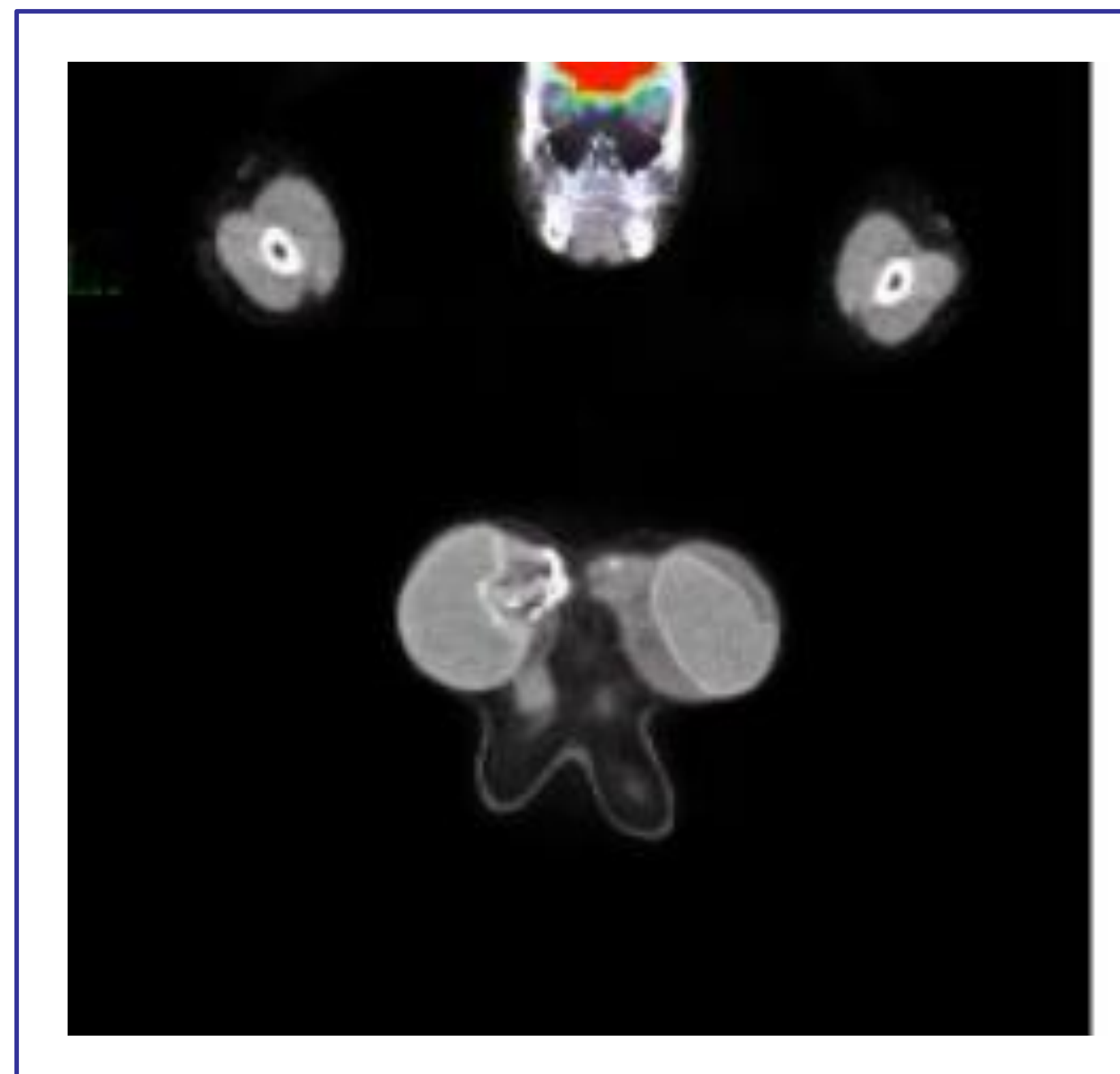

Pet-CT showed a localized disease with low standardized value in the fluid adjacent the left breast implant

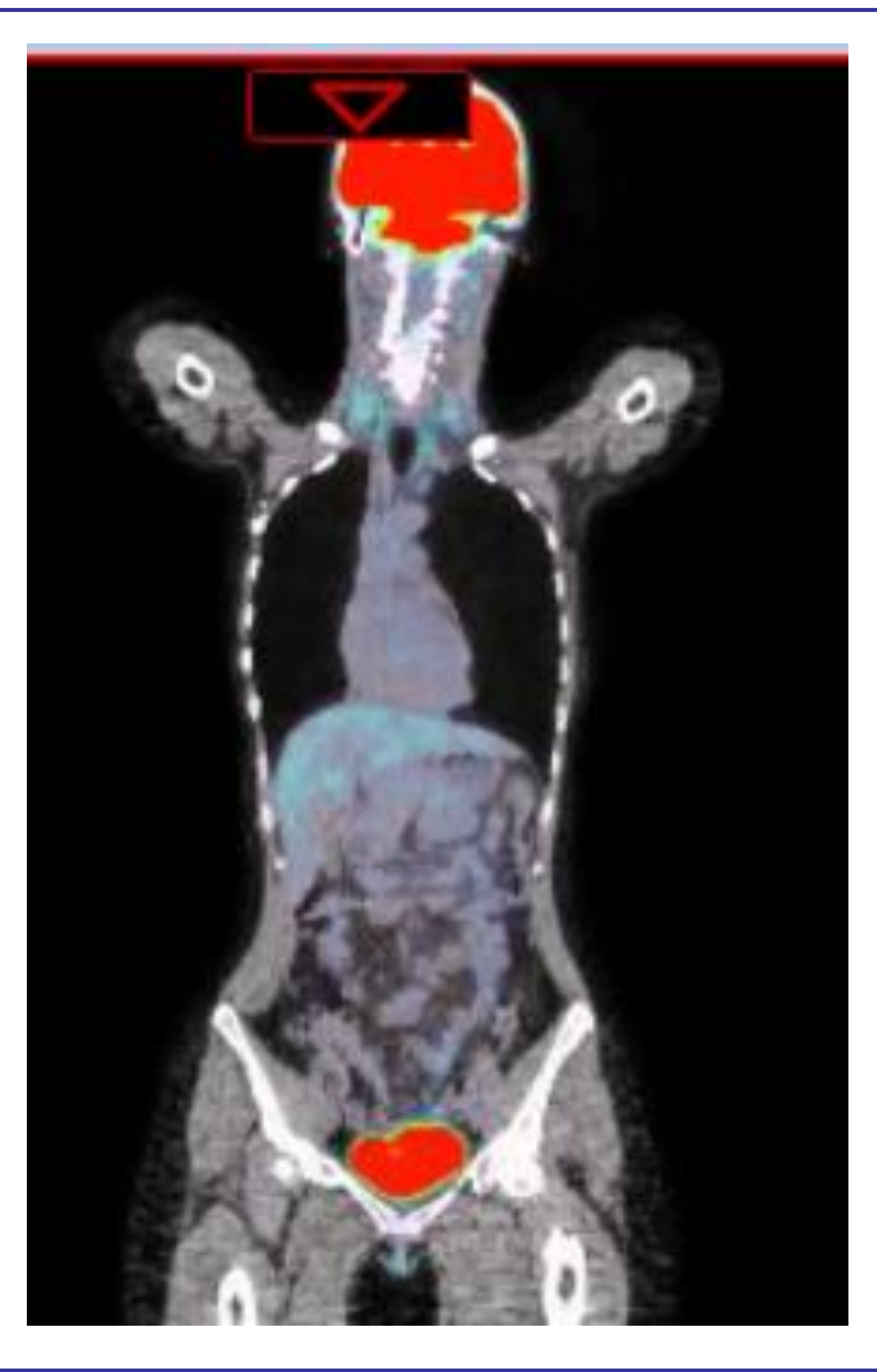

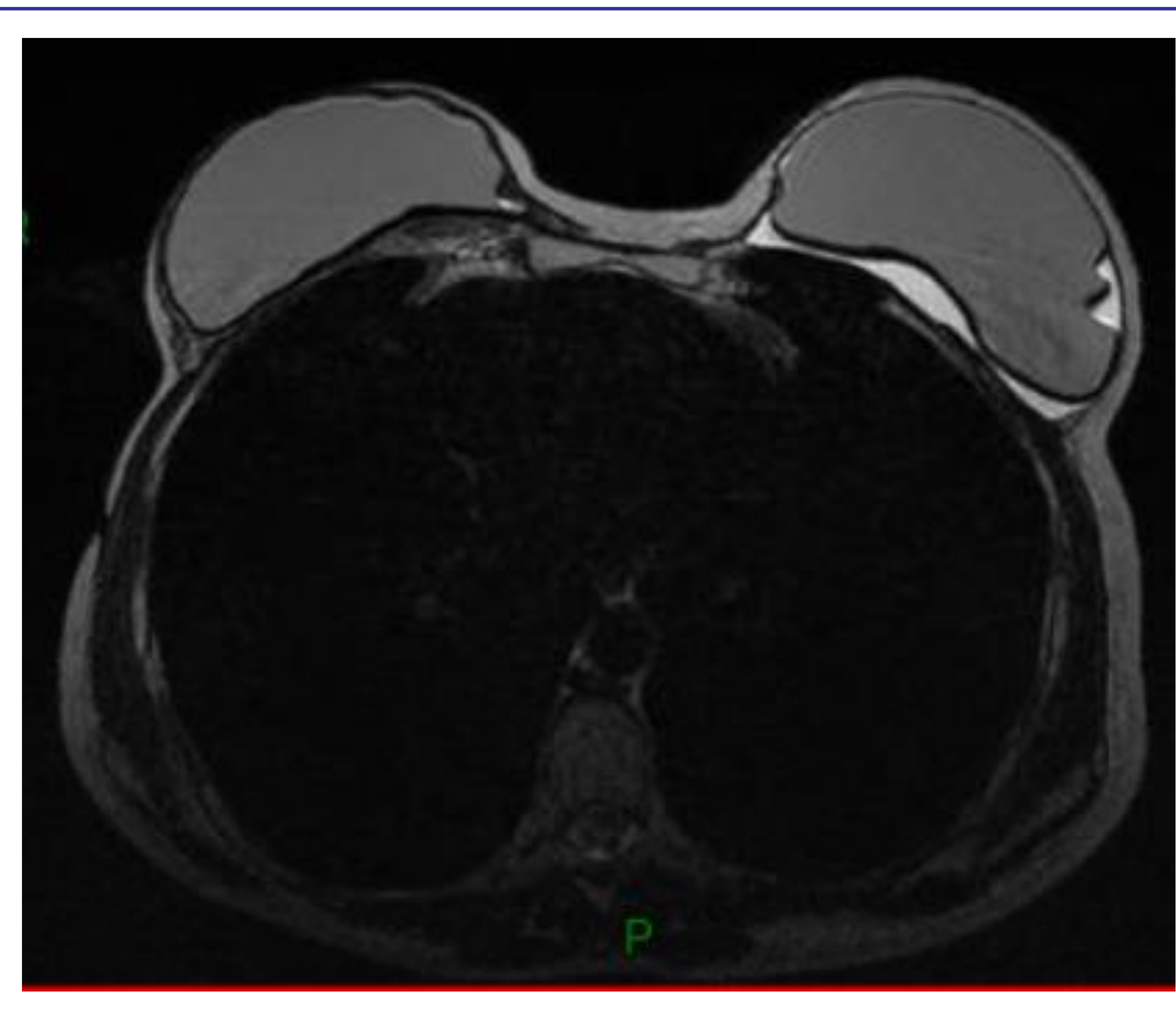

MRI showed periprosthetic fluid without any mass, and the implant was intact

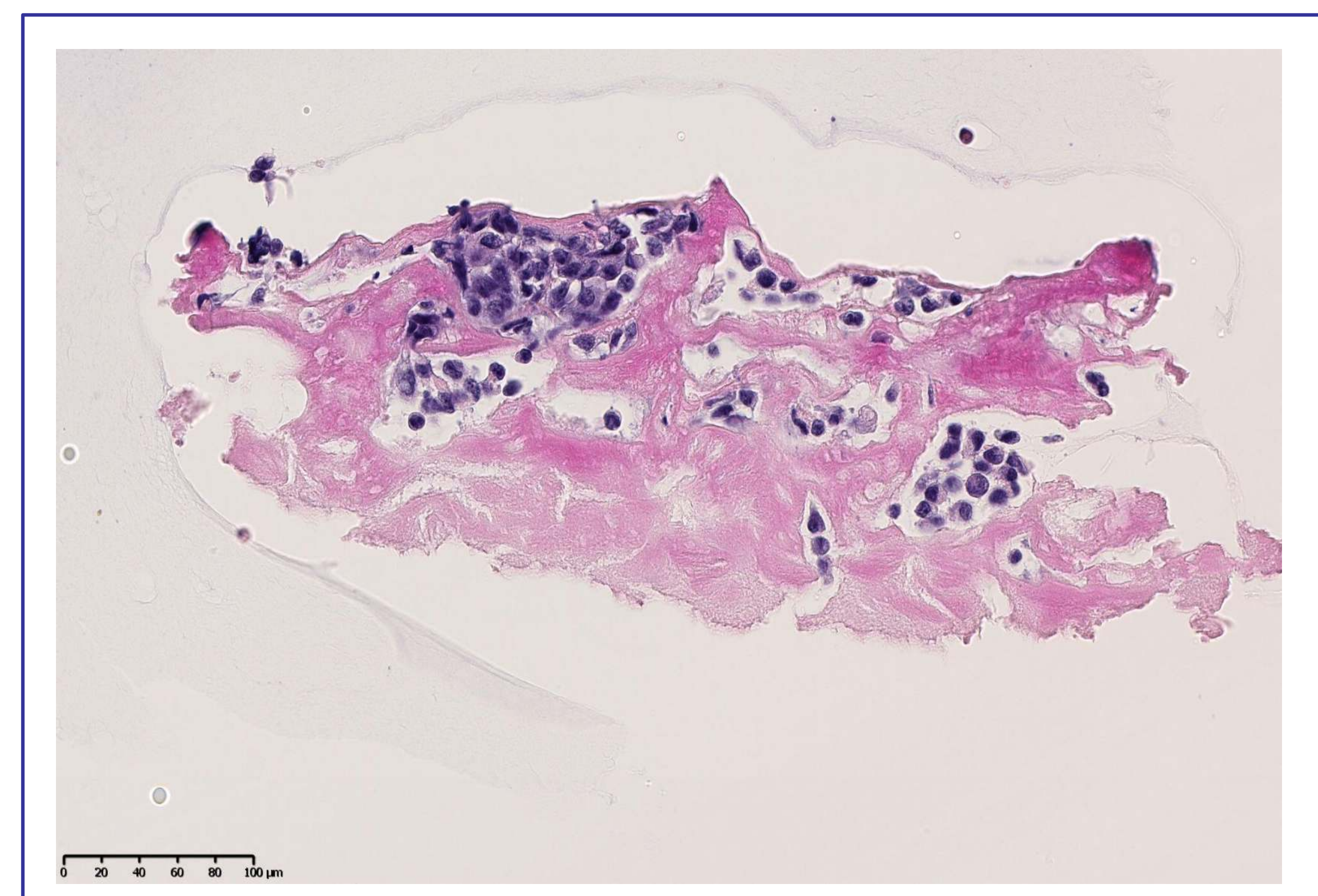

Cytology analysis of the aspirate fluid revealed an anaplastic large T-cell lymphoma

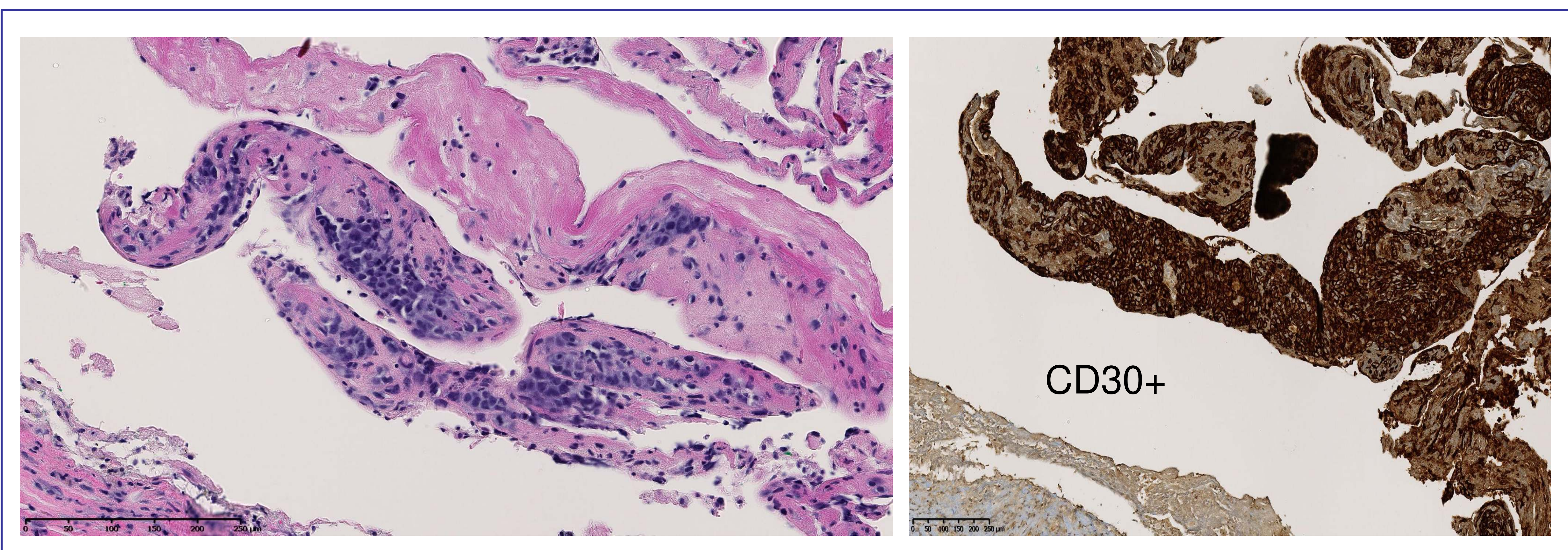

Bilateral total capsulectomy confirmed the diagnosis of anaplastic t-cell lymphoma, revealed as CD30positive, ALK 1-negative and confined to the fibrinoid material next to the prosthesis.

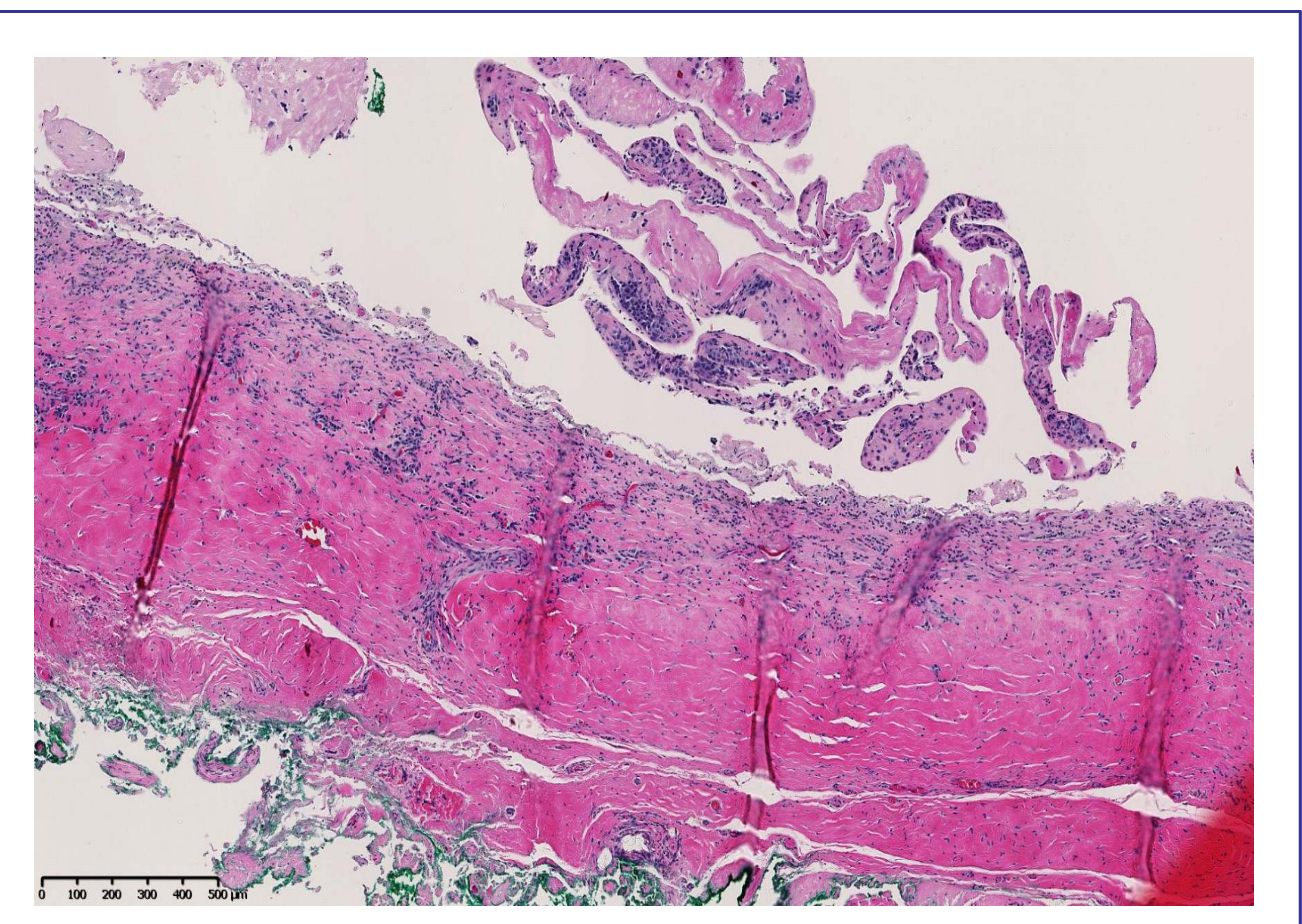

Fibrous capsule was tumour free

Breast implant anaplastic large T-cell lymphoma is a very rare cause of breast lymphoma. The treatment is surgery and the prognosis is really good if the tumour is localised by periprosthetic liquid. 